$<$ Original Article $>$

\title{
Disagreement between direct and indirect blood pressure measurements obtained from minipigs
}

\author{
Won-Jae Lee ${ }^{1}$, Lisa Soyeon Park ${ }^{2, *}$ \\ ${ }^{1}$ College of Veterinary Medicine, Kyungpook National University, Daegu 41566, Korea \\ ${ }^{2}$ Prestige BioResearch, Singapore 638117, Singapore
}

(Received: April 20, 2018; Revised: July 19, 2018; Accepted: August 2, 2018)

\begin{abstract}
Blood pressure (BP) measurement plays a pivotal role in veterinary medicine for diagnosing cardiovascular disorders and monitoring anesthesia of animals. Although indirect BP measurement has been widely applied to monitor $\mathrm{BP}$ because of its convenience and non-invasiveness, it is still unclear whether indirect BP measurement is compatible with direct BP measurement in minipigs. In addition, the effect of animal posture during BP measurement is not well understood in minipigs despite its importance to cardiovascular performance. Therefore, both systolic and diastolic arterial BPs in minipigs were measured via femoral artery catheterization for direct BP measurement and using a compressive cuff as an indirect BP measurement under the dorsal or right lateral recumbent postures. Numerical values were processed by the Bland-Altman method to calculate the bias $\pm \mathrm{SD}$ and the limits of agreement (LOA). In accordance with the American College of Veterinary Internal Medicine guidelines, the results between direct and indirect BP measurements were determined as apparent disagreements in both systolic and diastolic arterial BPs under all postures because of large bias \pm SD and wide LOA. The results of the present will help prevent misinterpretation of the anesthetized patient's condition during monitoring of BP by indirect measurement.
\end{abstract}

Keywords: anesthesia, blood pressure, diagnosis, miniature swine

\section{Introduction}

Because the primary role of the cardiovascular system is the supply of blood to whole organs, the blood pressure (BP) measurement is considered as a major indicator to evaluate its function [20]. Accurate BP measurement plays a pivotal role in the interpretation of the vascular biology, diagnosis of cardiovascular disease and hyper-/hypotensive patients, and monitoring of anesthetized patients [1]. The systolic arterial BP (SAP) indicates the pressure of the blood against the arterial wall when the heart contracts, and the diastolic arterial BP (DAP) implies the pressure in the artery while the heart is filling and resting between heart beats.

The two types of BP measurement methods, direct and indirect ones, have its unique advantages and disadvantages. Direct BP measurement is regarded as the gold standard of BP monitoring since it can provide reliable BP reporting, the most accurate results, and continuous measurements [23]. However, because direct BP measurement is an invasive procedure that accompanies intravascular catheterization in a suitable artery, it demands technical skill and may occur indispositions and complications to patients, such as ischemia in the distal region from a catheterized artery in case of long- term surgery $[1,23]$. In contrast, indirect BP measurement is generally addressed as a non-invasive arterial blood pressure (NIBP) measurement that requires the use of a compressive cuff; based on the oscillometric technique, the compressive cuff is usually applied externally to limbs to obstruct blood flow through the artery when it is inflated [2]. Because indirect BP measurement can be achieved by non-invasive procedure, it is preferable to direct BP measurements in veterinary medicine [15]. However, the accuracy of indirect BP measurements must be validated by means of comparison with direct BP measurements. Guidelines for validation of indirect $\mathrm{BP}$ measurement in the animals have been provided from the American College of Veterinary Internal Medicine (ACVIM), and have strongly suggested that the method of indirect BP measurement needs to be validated against direct BP measurement prior to its application to animals [2].

Meanwhile, the physical characteristics of minipigs with respect to shorter, thicker, and conically shaped legs may affect the application of a compressive cuff for indirect BP measurement, but the validation for agreement between direct and indirect BP measurement in minipigs is still limited. In addition, it was addressed that different postures in human during indirect BP measurement significantly affected the

*Corresponding author

Tel: +82-65-6863-3042, Fax: +82-65-6863-4834

E-mail: pwggeneticslisa@gmail.com 
cardiovascular performance, resulting in the variances of the numerical values of BP [8]. Although the effects of animal posture during $\mathrm{BP}$ measurement have been investigated in some animal species such as dogs, horses, and birds, the relevant study is not well investigated in the pigs [7, 11, 12]. Therefore, the present study was aimed at clarifying whether indirect BP measurement is compatible with direct BP measurement in minipigs under different postures (dorsal or right lateral recumbency), in accordance with the ACVIM guideline.

\section{Material and Methods}

\section{Ethics and animal}

All procedures regarding the preparation and operation of animals were approved by the Institutional Animal Care and Use Committee (IACUC No. NP14060). A total number of 32 healthy 18 to 36-month old minipigs, weighing $29.6 \pm$ $2.8 \mathrm{~kg}$ (Prestige Bioresearch, Singapore) were employed in the present study. The right hind limb was employed for measuring direct BP, and the left hind limb was used for measuring indirect BP. Numerical values from direct and indirect BP measurement were concurrently captured from each animal.

\section{Direct BP measurement}

Because the status and depth of anesthesia might affect the results of the present study, anesthetist or veterinary technician continuously monitored the anesthetized animals in terms of respiratory rate/rhythm (normal range, $8-18$ breath $/ \mathrm{min}$ ), oxygen saturation $\left(\mathrm{SpO}_{2}\right.$; normal range, $\left.95-100 \%\right)$, heart rate (normal range, 60-100 beat $/ \mathrm{min}$ ), color of mucous membrane at the gingiva (normal state, pink), and the rectal temperature (normal range, $37.5-39.0^{\circ} \mathrm{C}$ ), and controlled appropriate depth of anesthesia to all minipigs, in accordance with internal standard operation procedure (SOP). Each animal was sedated by intramuscular injection of ketamine $(10 \mathrm{mg} / \mathrm{kg})$ and xylazine $(2.5 \mathrm{mg} / \mathrm{kg})$, and was anesthetized using $2 \%$ isoflurane inhalation in oxygen through the endotracheal intubation in the dorsal recumbent posture. Intravascular access via the femoral artery was applied for direct BP measurement. In an attempt to search a suitable site for the cutaneous incision, the region where the medial saphenous artery disappeared into the fascia division of the sartorius and gracilis muscles in the right hind limb was manually palpated, following which a $5-\mathrm{cm}$ longitudinal cutaneous incision was made. The subcutaneous tissue was bluntly dissected until the gracilis and sartorius muscles were clearly observed (Fig. 1A). The fascia division between the sartorius and gracilis muscles was separated with a self-retaining tissue retractor, and subsequently the femoral artery was trimmed so as not to destroy the femoral nerve and vein (Fig. 1B). The exposed femoral artery was cannulated using an $18 \mathrm{G} \times 45 \mathrm{~mm}$ BD Venflon Pro Peripheral IV Catheter (BD, USA), after which the needle was discarded. The catheter was withdrawn upon the insertion of a 0.025 -inch $\mathrm{J}$ guide wire (Fixed Core J Tip Guidewires; Argon Medical Devices, USA) through the catheter. A $4 \mathrm{~F}$ catheter (Argon Medical Devices) was advanced over the $\mathrm{J}$ guide wire, and then the $\mathrm{J}$ guide wire was discarded. After checking whether a blood sample could be drawn through the catheter, $500 \mathrm{IU} / \mathrm{mL}$ of heparin mixed with isotonic saline was used as the catheter lock solution to prevent blood clots in the catheter. A TruWave Disposable Pressure Transducer Kit (Edwards Lifesciences, USA) filled with saline was placed between the $4 \mathrm{Fr}$ catheter and the patient monitor (Dräger, Germany) (Fig. 1C). All procedures related to the femoral artery catheterization were performed aseptically to avoid possible infections. The incision site was closed after obtaining numerical value of direct BP measurement. All animals were treated with administration of antibiotics (Baytril; Bayer Healthcare, Germany) for 3 days.

\section{Indirect BP measurement}

In order to collect numerical values of indirect BP mea-
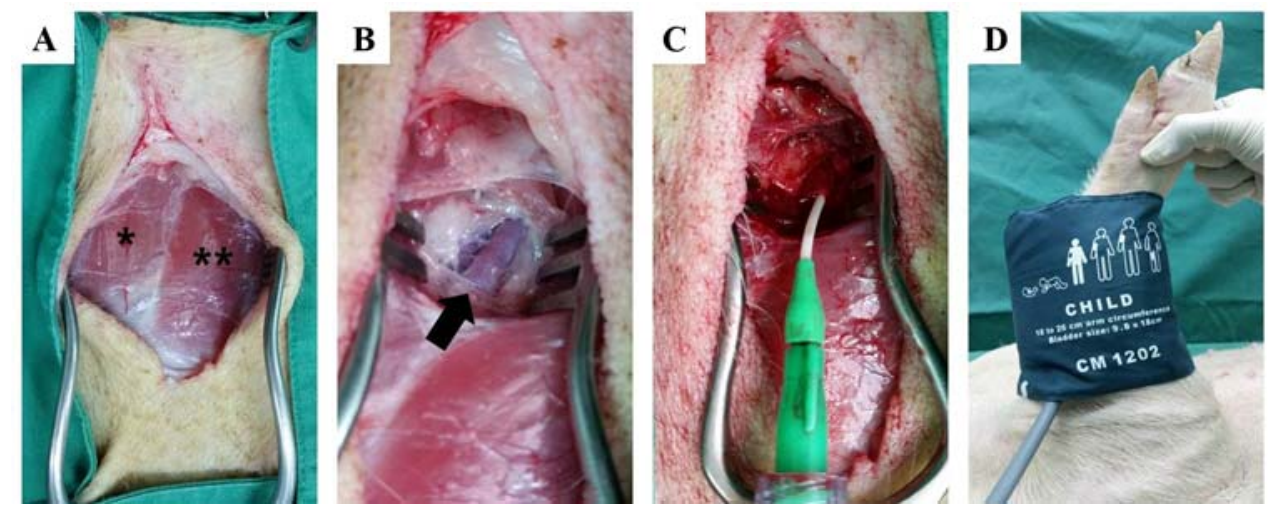

Fig. 1. Direct blood pressure (BP) measurement via the femoral catheterization and indirect BP measurement using a compressive cuff. (A) The subcutaneous tissue was bluntly dissected until the gracilis and sartorius muscles were clearly observed in the right hind limb. Asterisk or asterisks indicate the gracilis or sartorius muscles, respectively. (B) The arrow indicates the exposed femoral artery. (C) The intravascular catheterization was conducted via the femoral artery. (D) The compressive cuff was applied to the left hind limb for indirect BP measurement in the dorsal or lateral recumbent posture. 
surements, a child-sized cuff (Dräger) was applied to minipigs in accordance with the manufacturer's instructions. This type of cuff was recommended for use in children, who has a 16$26 \mathrm{~cm}$ arm circumference; it was the similar specification for the hind limb of a minipig. Once the cuffs was placed in the left hind limb of the minipig, the animal posture was maintained as the dorsal recumbent posture $(n=17)$ or repositioned to the right lateral recumbent posture $(n=15)$. After connecting the cuff tube to the NIBP port in the same patient monitor (Dräger), numerical value of indirect BP measurement was captured under a stably relaxed position of the hind limb (Fig. 1D). Both the SAP and DAP values from direct BP measurements were concurrently captured with those from indirect BP measurements on the patient monitor.

\section{Statistical analysis}

Turkey's range test (post hoc analysis) by PASW software (SPSS, USA) was used to analyze for significant differences in direct SAP (dSAP) measurements in dorsal (DP) and lateral (LP) recumbent postures (dSAP-DP and dSAP-LP), and those by indirect BP (iSAP) measurements (iSAP-DP and iSAP-LP). In addition, same analysis was concurrently conducted in direct DAP (dDAP) measurements in DP and LP (dDAP-DP and dDAP-LP), and those by indirect BP (iDAP) measurements (iDAP-DP and iDAP-LP). A $p$ value less than 0.05 was considered to be significant. The Bland-Altman method was used to evaluate the agreement between direct and indirect BP measurements; the mean difference (bias) \pm $\mathrm{SD}$ as well as the limits of agreement (LOA) was calculated. In this analysis, the difference $(\mathrm{A}-\mathrm{B})$ between method $\mathrm{A}$ (direct BP measurement) and method B (indirect BP measurement) was compared with the mean of the two methods (bias, $(\mathrm{A}+\mathrm{B}) / 2$ ) [9]. In accordance with the ACVIM guideline, the agreement was determined when the bias or its SD was within \pm 10 or $\pm 15 \mathrm{mmHg}$, respectively [2].

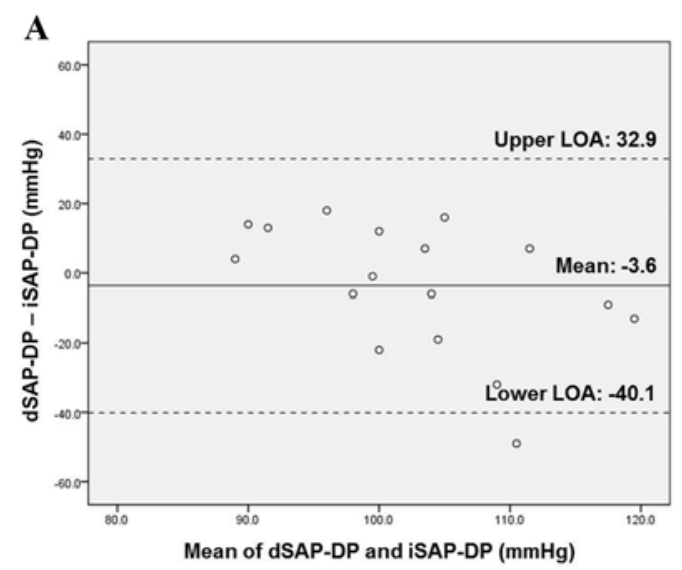

\section{Results}

\section{Normal level of SAP and DAP in minipigs in the dif- ferent postures}

The numerical values of the SAP and DAP, obtained from both direct BP measurement via the femoral artery catheterization and indirect BP measurements using a compressive cuff, were compared in figure 2 . The numerical values in SAP by direct and indirect BP measurements under different postures of minipigs ranged from $98.7 \pm 10.3 \mathrm{mmHg}$ (dSAP$\mathrm{LP}$ ) to $104.8 \pm 15.7 \mathrm{mmHg}$ (iSAP-DP) without presenting the significant difference. In contrast, a significantly $(p<0.05)$ lower DAP was exhibited in indirect BP measurements under both postures in comparison with those of direct BP measurements; the normal level of the DAP in minipigs was considered to be from $72.7 \pm 5.3 \mathrm{mmHg}$ (dDAP-LP) to $74.0 \pm$

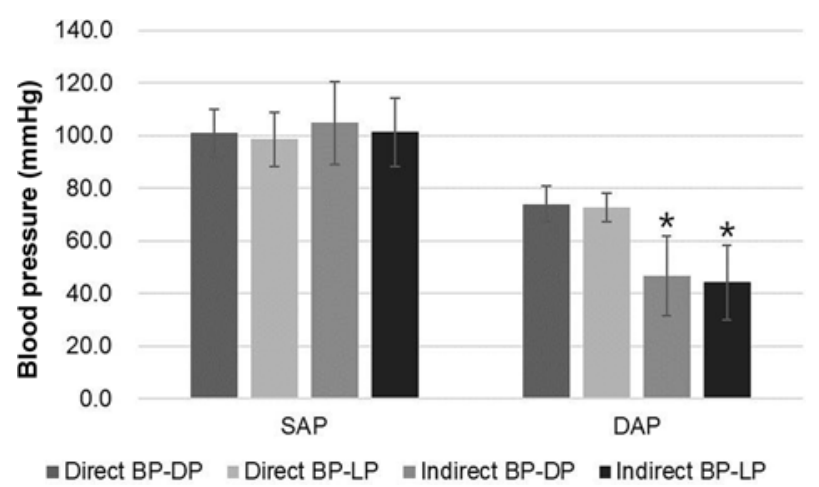

Fig. 2. Normal level of the systolic arterial BP (SAP) and diastolic arterial BP (DAP) in minipigs in the different postures. The numerical values in the bars indicated means of the SAP and DAP $(\mathrm{mmHg})$ in direct and indirect BP measurements in the different postures. Significant $(p<0.05)$ differences were denoted with asterisk. DP, dorsal recumbent posture; LP, lateral recumbent posture.

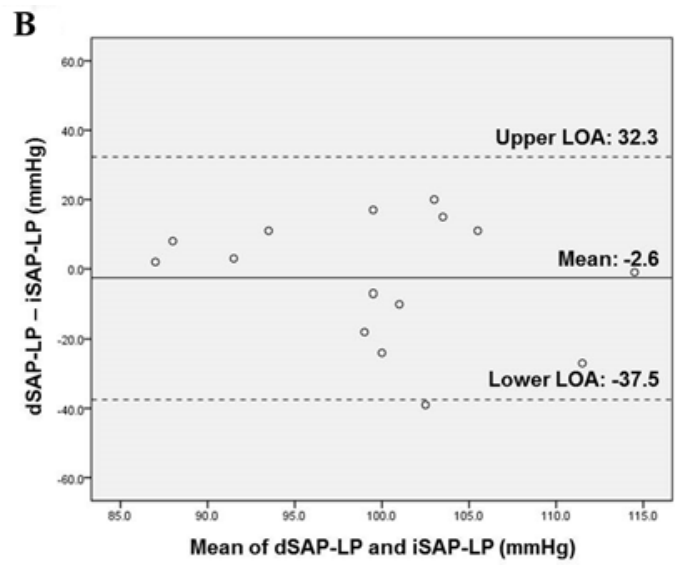

Fig. 3. Validation of the agreement between direct and indirect BP measurements in the SAP in the dorsal (A) or lateral (B) recumbent posture. Bland-Altman plots of the agreement in the SAP between direct and indirect BP measurement were analyzed. A solid line or two dotted lines indicated the bias or the limits of agreement (LOA), respectively. dSAP-DP, direct SAP measurement in DP; dSAPLP, direct SAP measurement in LP; iSAP-DP, indirect SAP measurement in DP; iSAP-LP, indirect SAP measurement in LP. 
A

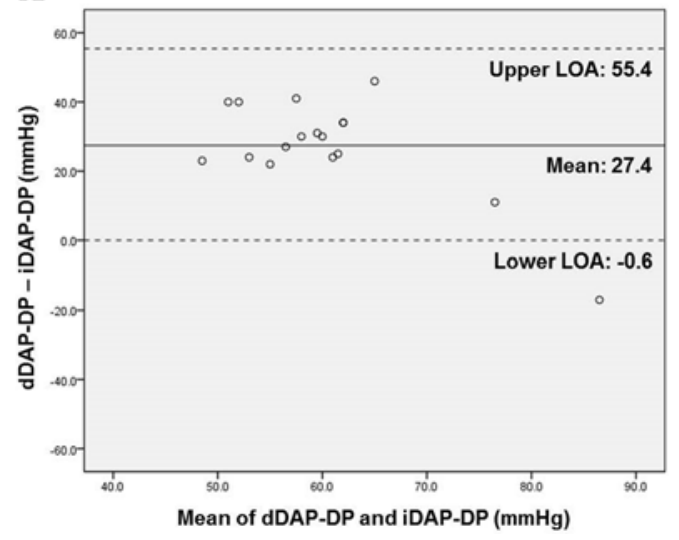

B

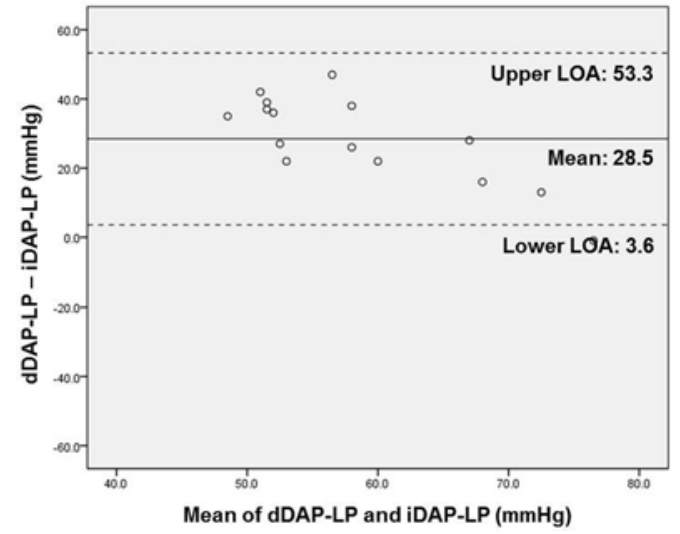

Fig. 4. Validation of the agreement between direct and indirect BP measurements in the DAP in the dorsal (A) or lateral (B) recumbent posture. Bland-Altman plots of the agreement in the DAP between direct and indirect BP measurement were analyzed. A solid line or two dotted lines indicated the bias or LOA, respectively.

6.7 mmHg (dDAP-DP) from direct BP measurement, but indirect BP measurement presented an approximately $0.62-$ fold lesser numerical value from $44.2 \pm 14.2 \mathrm{mmHg}$ (iDAP$\mathrm{LP}$ ) to $46.7 \pm 15.2 \mathrm{mmHg}$ (iDAP-DP). Overall, there was no significant change depending on the different postures in each SAP and DAP.

\section{Agreement between direct and indirect BP measurement}

All numerical values of the SAP and DAP by both direct and indirect $\mathrm{BP}$ measurements under different postures were processed in accordance with Bland-Altman method, and representative plots were generated to evaluate the bias $\pm \mathrm{SD}$ as well as LOA (Figs. 3 and 4). The results for the SAP in the dorsal recumbent posture (dSAP-DP and iSAP-DP) showed a $-3.6 \pm 18.6 \mathrm{mmHg}$ as the bias $\pm \mathrm{SD}$, and the LOA was determined to range from -40.1 to $32.9 \mathrm{mmHg}$ (Fig. 3A). Therefore, the agreement between direct and indirect BP measurement for the SAP in the dorsal recumbent posture was rejected due to higher values of the SD in accordance with the ACVIM guideline. The bias $\pm \mathrm{SD}$ in the DAP measurement in the dorsal recumbent posture (dDAP-DP and iDAP-DP) was $27.4 \pm 14.3 \mathrm{mmHg}$, with the LOA ranging from -0.6 to 55.4 $\mathrm{mmHg}$ (Fig. 4A). Hence, monitoring the DAP in the dorsal recumbent posture by indirect BP measurement was also disagreed because the bias did not meet the ACVIM guideline. By the same token, both SAP (bias \pm SD from dSAP-LP with iSAP-LP: $-2.6 \pm 17.8 \mathrm{mmHg}$ ) and DAP (bias \pm SD from dDAPLP with iDAP-LP: $28.5 \pm 12.7 \mathrm{mmHg}$ ) in the lateral recumbent posture were also disagreed (Fig. 3B and 4B). Consequentially, it was identified that BP monitoring by means of indirect BP measurement in minipigs was not acceptable in any postures, in accordance with the ACVIM guideline.

\section{Discussion}

Various kinds of methods for BP measurements have been established for several decades [22]. BP monitoring is widely employed in the diagnosis of the cardiovascular disease, and for checking vital signs during anesthesia. The risk of anesthesia-related death due to the cardiovascular system complications is $0.05 \%$ or $1.33 \%$ in healthy or sick dogs, respectively. Therefore, monitoring of the cardiovascular system, especially for hypotension, is essential during anesthesia [4]. Indirect $\mathrm{BP}$ measurement has been widely applied to monitor $\mathrm{BP}$ in animals due to its convenience and non-invasiveness. However, in agreement of ACVIM guideline, validation of indirect BP measurement method is necessary to prevent misinterpretation of the anesthetized patient's condition [2, 3]. Therefore, the present study focused on the validation of the agreement between direct and indirect BP measurements in the minipigs under different postures, in accordance with ACVIM guideline.

It is known that many factors including the posture influence to indirect BP measurement. When BP was indirectly measured in humans with different positions such as sitting, standing, and supine postures, both SAP and DAP were higher in supine position than those of others [8]. In addition, indirect measurements of SAP were affected as higher for dogs when sitting rather than laterally recumbent [14]. Therefore, the comparative studies for validation of indirect BP measurement between different postures in minipigs were necessary to be conducted, because these studies were still limited in pigs; in most cases, BP measurement in a single dorsal recumbent posture was mainly investigated in pigs in the previous studies $[10,13,21]$. Overall, it was hard to find the considerable effect between the value of BP and animal posture in the present study (Fig. 2). In agreement with the present study, BP measurements in anesthetized dogs, horses, and birds also exhibited no considerable variances depending on the different postures [7, 11, 12]. Based on these results, it can be concluded that the animal posture in the anesthetized status less affects to BP and the cardiovascular 
performance, possibly due to unconscious condition for the external stimuli, relaxed posture which is not sufficient impact to reduce venous return into the heart, and similar height level between BP measured site and the heart when the animals are lain on the surgical table.

When pigs are anesthetized by inhalant, they can be positioned as dorsal, ventral, and lateral recumbent posture during endotracheal intubation. Whereas placement of endotracheal tube in ventral recumbent posture has difficulty in pulling the epiglottis down from its position overlying the soft palate, that of dorsal recumbent posture has the merits of unnecessity of assistance to open the jaws, easiness in pigs less than $50 \mathrm{~kg}$, and shorter time for safe intubation $[16,19]$. In addition, pigs are commonly employed as animal model for preclinical surgical studies due to their similarities with humans in anatomy, cardiovascular system, and gastrointestinal tract; dorsal recumbent posture is recommended in laparotomyrelated and articular studies, and lateral recumbent posture is preferable to cardiovascular, ophthalmology, and odontological researches $[13,17,18]$. Therefore, we selected two postures (dorsal and lateral recumbent postures) in the anesthetized minipigs to validate agreement between direct and indirect BP measurement in the present study, and demonstrated the low accuracy and reproducibility of indirect BP measurement in minipigs in the dorsal and lateral recumbent postures, which could occur misinterpretation of condition of the anesthetized minipigs during preclinical surgical studies.

The investigation for application of indirect BP measurement in the domestic or farm pig model have been explored; indirect BP measurement methods by means of automated oscillometry or Doppler probe or NIBP cuff in the farm pigs showed the disagreement or great difference between direct and indirect BP measurement [5, 10, 13, 21]. In case of minipig breed, validation of indirect BP measurement has been reported in only a few articles; application of a photoelectric flow sensor (indirect BP measurement) to the tails of minipigs presented that DAP and mean arterial pressure (MAP) from indirect BP measurement were consistently underestimated in comparison with those from direct BP measurement [6]. Based on the aforementioned articles, it can be concluded that the validation of indirect BP measurement method in minipigs in the different postures is necessary to prevent the predicted misinterpretation of the patient's condition during the diagnosis and monitoring anesthesia. The present study determined a wide level of the disagreement between direct and indirect BP measurement in minipigs under different postures. In the present study, all numerical values of the SAP and DAP obtained by both direct and indirect $\mathrm{BP}$ measurements were processed in accordance with Bland-Altman method to calculate the bias, SD and LOA. In accordance with the ACVIM guideline, the agreement can be defined only if the value of the bias or SD is within range of \pm 10 or $\pm 15 \mathrm{mmHg}$, respectively [2]. The values of the bias of the SAP in the dorsal recumbent posture $(-3.6 \mathrm{mmHg})$ and the lateral recumbent posture $(-2.6 \mathrm{mmHg})$ were deter- mined as satisfaction with the ACVIM guideline. However, both the SD and the LOA values were too large to be accepted as the agreement in accordance with the ACVIM guideline; in particular, SDs $(18.6 \mathrm{mmHg}$ in the dorsal recumbent posture and $17.8 \mathrm{mmHg}$ in the lateral recumbent posture) were out of the range of the ACVIM guideline (Fig. 3). In case of the DAP, indirect BP measurement was disagreed due to excessive values of the bias $(27.4 \mathrm{mmHg}$ in the dorsal recumbent posture and $28.5 \mathrm{mmHg}$ in the lateral recumbent posture) even though the value of SDs were placed within the range of the ACVIM guideline (Fig. 4).

Direct and indirect BP measurements are basically different from each other; direct BP measurement detects blood pressure in the vessel at first hand, on the contrary, indirect BP measurement estimates BP using blood flow-induced pressure oscillations through the compressive cuff. Therefore, a variety of factors may potentially affect the reproducibility and variability of indirect BP measurement. In the previous study, possible sources of variability were attributed to inconsistency aspects in the equipment and the patients [3]. The primary errors were potentially caused by equipment with respect to improper cuff size, residual air in the cuff, a loose cuff, and different tubing sizes and lengths. Variations in patients that can influence BP measurements included shock, poor ventilation, dysrhythmias, and differences in anatomy. Of particular, the anatomy of minipigs can affect numerical values in indirect BP measurement; because they have shorter, thicker, and conically shaped legs, poor delivery of pressure to the air within the cuff can cause the short and weak pulse wave and an underestimation of the arterial pressure.

In conclusion, the results of the present study determined that apparent disagreement existed between direct and indirect $\mathrm{BP}$ measurements in minipigs. The low accuracy and reproducibility of indirect $\mathrm{BP}$ measurement in minipigs were determined by a large bias and wide LOA. Even though direct BP measurement is currently employed in veterinary medicine and in preclinical studies, indirect BP measurement is also widely applied without appropriate validation. Therefore, it is necessary to address the importance of validation of routinely used BP measurement devices to avoid misinterpretation of the patient's condition during the diagnosis and monitoring anesthesia.

\section{Acknowledgments}

This work was supported by a grant from the National Research Foundation (NRF) of Korea, funded by the government of the Republic of Korea (Grant No. NRF-2017 R1C1B5076029), and was conducted with Prestige BioResearch (Singapore 638117).

\section{References}

1. Acierno MJ, da Cunha A, Smith J, Tully TN Jr, Guzman 
DSM, Serra V, Mitchell MA. Agreement between direct and indirect blood pressure measurements obtained from anesthetized Hispaniolan Amazon parrots. J Am Vet Med Assoc 2008, 233, 1587-1590.

2. Almeida D, Barletta M, Mathews L, Graham L, Quandt J. Comparison between invasive blood pressure and a noninvasive blood pressure monitor in anesthetized sheep. Res Vet Sci 2014, 97, 582-586.

3. Bass LME, Yu DY, Cullen LK. Comparison of femoral and auricular arterial blood pressure monitoring in pigs. Vet Anaesth Analg 2009, 36, 457-463.

4. Brodbelt DC, Blissitt KJ, Hammond RA, Neath PJ, Young LE, Pfeiffer DU, Wood JLN. The risk of death: the confidential enquiry into perioperative small animal fatalities. Vet Anaesth Analg 2008, 35, 365-373.

5. Chow PKH, Ng TH, Heng D, Mack POP. A simple method of blood pressure measurement in the pig using a neonatal cuff. Ann Acad Med Singapore 1999, 28, 15-19.

6. Cimini CM, Zambraski EJ. Non-invasive blood pressure measurement in Yucatan miniature swine using tail cuff sphygmomanometry. Lab Anim Sci 1985, 35, 412-416.

7. Drynan EA, Schier M, Raisis AL. Comparison of invasive and noninvasive blood pressure measurements in anaesthetized horses using the Surgivet V9203. Vet Anaesth Analg 2016, 43, 301-308.

8. Eșer I, Khorshid L, Güneș UY, Demir Y. The effect of different body positions on blood pressure. J Clin Nurs 2007, 16, 137-140.

9. Giavarina D. Understanding Bland Altman analysis. Biochem Med (Zagreb) 2015, 25, 141-151.

10. Gladczak AK, Shires PK, Stevens KA, Clymer JW. Comparison of indirect and direct blood pressure monitoring in normotensive swine. Res Vet Sci 2013, 95, 699-702.

11. Goya S, Wada T, Shimada K, Hirao D, Fukushima R, Yamagishi N, Shimizu M, Tanaka R. Effects of postural change on transesophageal echocardiography views and parameters in healthy dogs. J Vet Med Sci 2017, 79, 380386.

12. Hawkins MG, Malka S, Pascoe PJ, Solano AM, Kass PH, Ohmura H, Jones JH. Evaluation of the effects of dorsal versus lateral recumbency on the cardiopulmonary system during anesthesia with isoflurane in red-tailed hawks (Buteo jamaicensis). Am J Vet Res, 2013, 74, 136-143.

13. Musk GC, Costa RS, Tuke J. Doppler blood pressure measurement in pigs during anaesthesia. Res Vet Sci 2014, 97, 129-131.

14. Rondeau DA, Mackalonis ME, Hess RS. Effect of body position on indirect measurement of systolic arterial blood pressure in dogs. J Am Vet Med Assoc 2013, 242, 15231527.

15. Stepien RL, Rapoport GS, Henik RA, Wenholz L, Thomas CB. Comparative diagnostic test characteristics of oscillometric and Doppler ultrasonographic methods in the detection of systolic hypertension in dogs. J Vet Intern Med 2003, 17, 65-72.

16. Swindle MM. Swine in the Laboratory: Surgery, Anesthesia, Imaging, and Experimental Techniques. 2nd ed. pp. 40-42, CRC, Boca Raton, 2007.

17. Swindle MM, Makin A, Herron AJ, Clubb FJ Jr, Frazier KS. Swine as models in biomedical research and toxicology testing. Vet Pathol 2012, 49, 344-356.

18. Swindle MM, Nolan T, Jacobson A, Wolf P, Dalton MJ, Smith AC. Vascular access port (VAP) usage in large animal species. Contemp Top Lab Anim Sci 2005, 44, 7-17.

19. Theisen MM, Maas M, Hartlage MAG, Ploner F, Niehues SM, Van Aken HK, Weber TP, Unger JK. Ventral recumbency is crucial for fast and safe orotracheal intubation in laboratory swine. Lab Anim 2009, 43, 96-101.

20. Thiele RH, Durieux ME. Arterial waveform analysis for the anesthesiologist: past, present, and future concepts. Anesth Analg 2011, 113, 766-776.

21. Tuohy PP, Raisis AL, Drynan EA. Agreement of invasive and non-invasive blood pressure measurements in anaesthetised pigs using the Surgivet V9203. Res Vet Sci 2017, 115, 250254.

22. Ward M, Langton JA. Blood pressure measurement. Contin Educ Anaesth Crit Care Pain 2007, 7, 122-126.

23. Wang Y, Cong Y, Li J, Li X, Li B, Qi S. Comparison of invasive blood pressure measurements from the caudal ventral artery and the femoral artery in male adult SD and Wistar rats. PLoS One 2013, 8, e60625. 